Supporting information

\title{
Metasurface-Integrated Photonic Platform for Versatile Free-Space Beam Projection with Polarization Control
}

Alexander Yulaev, ${ }^{1,2}$ Wenqi Zhu, ${ }^{1,2}$ Cheng Zhang, ${ }^{1,2}$ Daron A. Westly, ${ }^{1}$ Henri J. Lezec, ${ }^{1}$ Amit Agrawal, ${ }^{1,2}$ and Vladimir Aksyuk ${ }^{1, *}$

${ }^{1}$ Physical Measurement Laboratory, National Institute of Standards and Technology, Gaithersburg, MD 20899, USA

${ }^{2}$ Maryland Nanocenter, University of Maryland, College Park, MD 20742 USA

7 pages

6 Figures 
Characterization of a free-space beam emanated from an extreme mode converter (EMC):

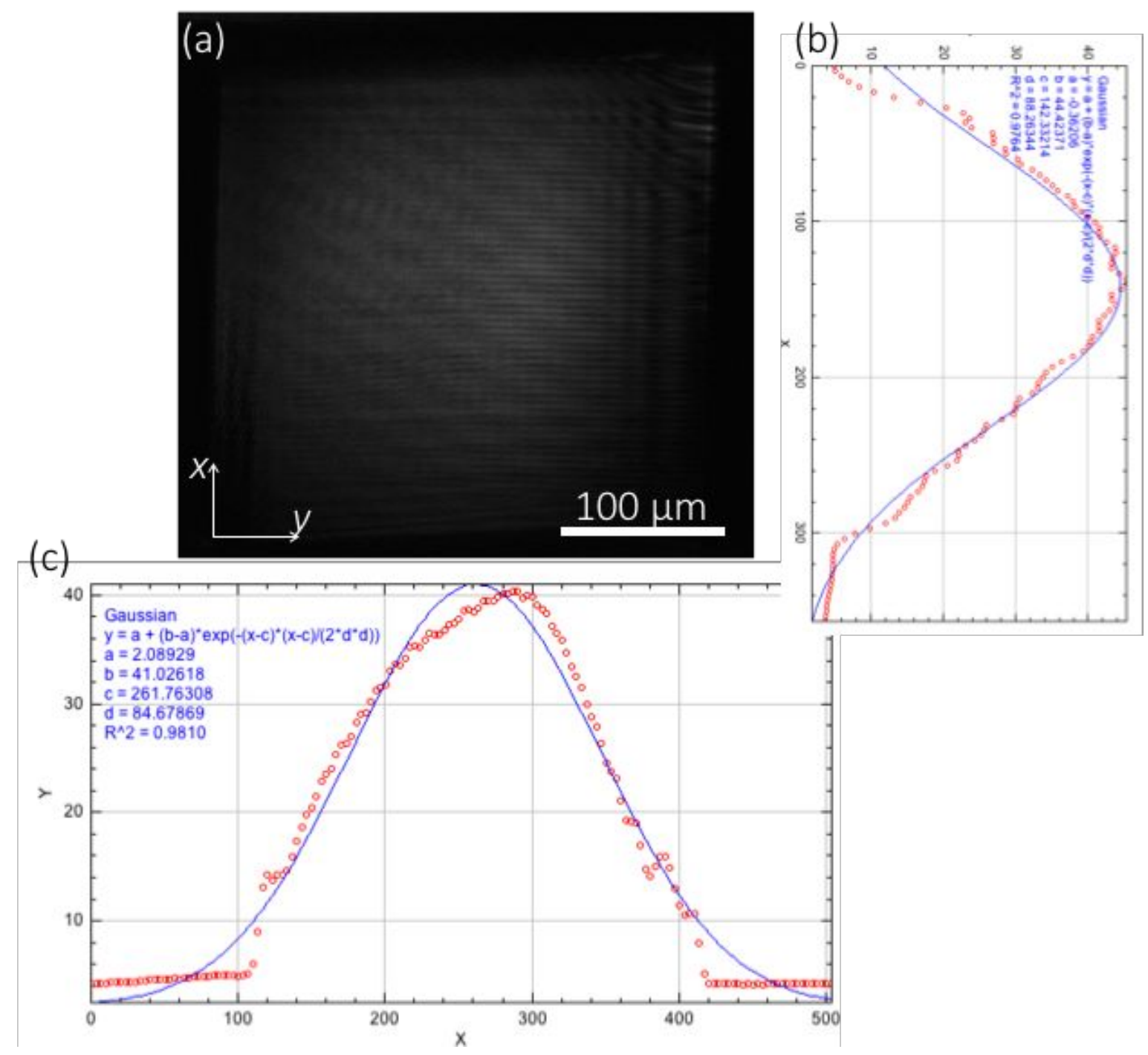

Fig. S1. Characterization of a free-space beam generated by an extreme mode converter. (a) A microscope micrograph of a free-space Gaussian beam projected by a $300 \mu \mathrm{m} \times 300 \mu \mathrm{m}$ grating of an extreme mode converter. (b)-(c) Mode profiles along $x$ and $y$ axes obtained by integrating light intensity along $y$ and $x$ directions, respectively. Fitting the experimental profiles with a 1D Gaussian function reveals $208 \mu \mathrm{m}$ and $199 \mu \mathrm{m}$ full-width at half-maximum (FWHM) along the $x$ and $y$ axes, respectively. 


\section{Effect of metasurface (MS) phase compensation on a focal spot:}

To analyze how the incorporated phase compensation affects the focal image, we fabricate and analyze an array of MS samples with a variety of angle correction values ranging from $0^{\circ}$ to $6.5^{\circ}$ in free space using Eq. (1) (Fig. S2). The left image in top row panel (a) shows the simulated $E_{x^{-}}$ field distribution after passing the $5^{\circ}$ phase-compensated MS. The other images in Fig. S2 depict the experimental focal spots projected from several phase-compensated MSs assembled and precisely aligned on top of the EMC. The in-plane projection $x^{\prime}$ of the light beam direction is marked with a dashed white arrow in Fig. S2. When the phase compensation of the MS is not properly tuned for the incident beam angle, the focal spot distorts, appearing as a coma aberration. For a given photonic chip, only $4.3^{\circ}$ phase-compensated MS results in an aberration-free focal spot image, which agrees well with the value of the beam tilt quantified from Fig. 3 b.
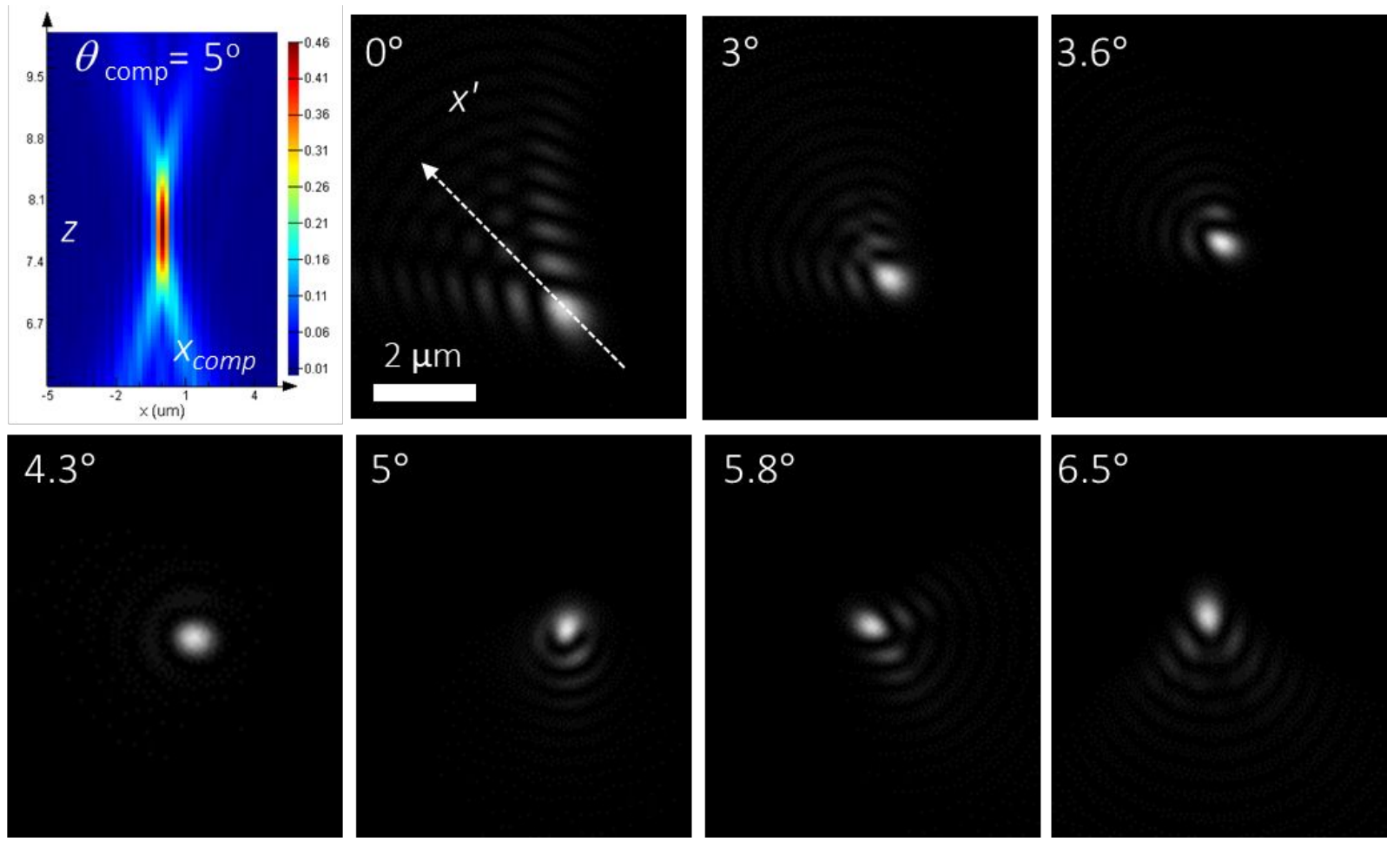

Fig. S2. Effect of phase compensation on focal spot aberration. The left image in top row is the longitudinal cross-section through a focused beam showing the simulated $E_{x}$-field distribution after passing $5^{\circ}$ phase-compensated metalens along the $x_{\text {comp }}$ direction. The other images acquired using $100 \times$ objective depict the experimental focal spots projected from $0^{\circ}$ to $6.5^{\circ}$ phase-compensated metalenses assembled and precisely aligned on top of the mode converter. The in-plane projection $x$ ' of the tilted incident light beam is marked with a dashed white arrow. 


\section{Effect of rotational misalignment on a focal spot:}

An adjustable mechanical contact between the MS and the EMC allows for facile and precise alignment resulting in an aberration-free image of the focal spot. However, integrating photonic chips with other systems may require the monolithic fabrication or assembling of the MS and the mode expander into a single unit. Because the exact EMC beam direction may be affected by fabrication process variation, quantifying the engineering tolerance to rotational misalignments is of high importance. Fig. S3 illustrates how focal spots distort when the rotational misalignment between the MS and the photonic chip is introduced. The misalignment angle $\Delta \theta$ is defined between the in-plane projection $x^{\prime}$ of the collimated free-space beam and the direction of the $4.3^{\circ}$ phase compensation $x_{\text {comp }}$ (Fig. S2 a). Optical images of the focal spots with $\Delta \theta=-2.8^{\circ}, 1.3^{\circ}$ result in an axially symmetric non-distorted focal spot (Fig.S3 b and c). However, once the $\Delta \theta$ grows to $\approx 10^{\circ}$, the focal spot undergoes deformation with a clearly defined coma-shaped tail (Fig. S3 d). The orientation of coma aberration qualitatively coincides with an in-plane projection of the mismatch between the $k$-vectors related to a free-space beam and a phase-compensated direction. To sum up, the MS design is required for a specific incident $k$-vector to achieve clean and aberration-free focusing, however the focusing quality is tolerant to $>3^{\circ}$ azimuthal angle variation.
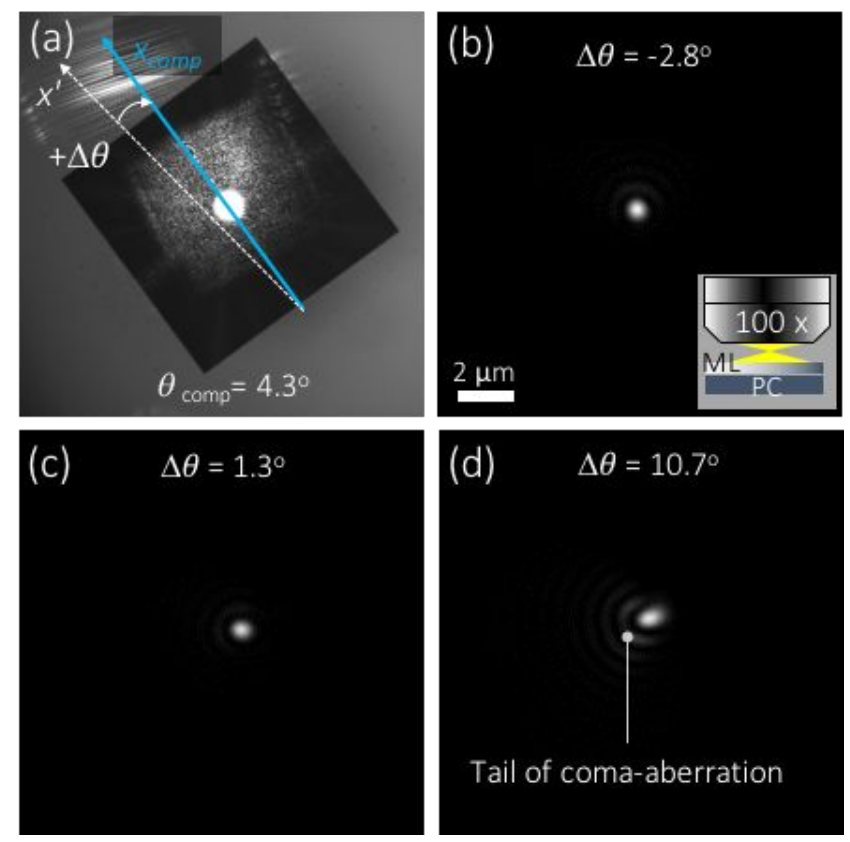

Fig. S3. Effect of rotational misalignment on focal spot aberration. (a) An optical image of the $4.3^{\circ}$ angle compensated metalens stacked with the mode converter captured using a $10 \times$ objective. $\Delta \theta$ defines the rotational misalignment angle between the in-plane projection $x^{\prime}$ of the collimated freespace beam and the direction of the $4.3^{\circ}$ phase compensation $x_{\text {comp }}$ incorporated into the metalens design. (b)-(d) Optical images of the focal spots obtained using $100 \times$ objective from a $4.3^{\circ}$ angle compensated metalens $(\mathrm{NA}=0.8)$ for $\Delta \theta=-2.8^{\circ}, 1.3^{\circ}$, and $10.7^{\circ}$. 

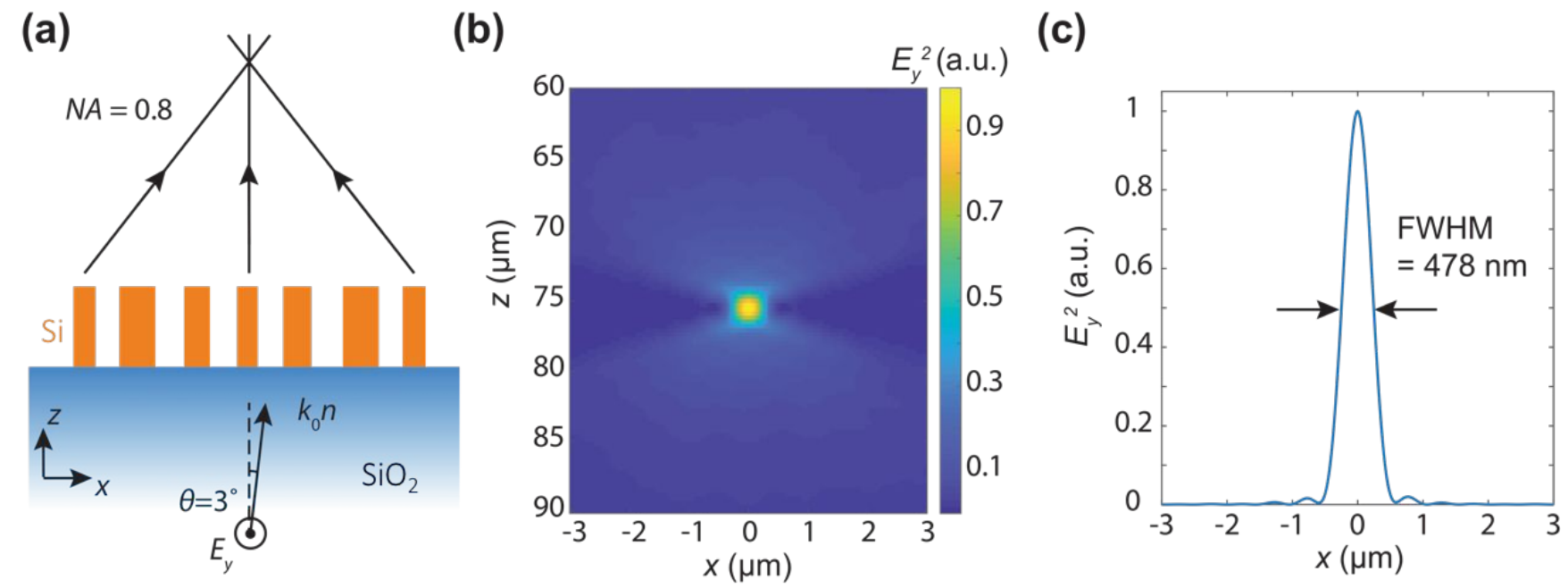

Fig. S4. Simulation of the metasurface that achieves focusing and tilt correction at the same time. (a) Schematic diagram of the metasurface functionality for an oblique angle of incidence $\boldsymbol{\theta}=\mathbf{3}^{\circ}$ in a silica substrate. The incident beam has a Gaussian profile with a $195 \mu \mathrm{m}$ FWHM. (b) The $x z-$ plane cross section of the FDTD simulated $\boldsymbol{E}_{\boldsymbol{y}}$ intensity at the vicinity of the focal spot. (c) Intensity profile of a focal spot along the $x$-axis. The simulated FWHM is $478 \mathrm{~nm}$.

We use three-dimensional finite-difference time-domain (FDTD) simulations to validate the designing concept of the metasurface that achieves focusing and tilt correction at the same time. The nanopillar array that constitutes the metasurface spans $20 \mu \mathrm{m}$ along the $x$-axis, where the inplane dimensions are set to implement the phase function of a cylindrical lens:

$$
\varphi(x)=\frac{2 \pi}{\lambda_{0}}\left(f-\sqrt{x^{2}+f^{2}}\right)-\frac{2 \pi}{\lambda_{0}} n x \sin \theta,
$$

where $\lambda_{0}=780 \mathrm{~nm}$ is the wavelength of incident light in free space, $f$ is the targeted focal length of the metalens, $n$ is the refractive index of the fused silica substrate, and $\theta$ is the angle of the obliquely incident light in the substrate. We have designed and simulated metalenses with targeted $f=7.8 \mu \mathrm{m}$ (corresponding to NA $=0.8$ ) for $\theta=3^{\circ}$ (Fig. S4 a-c). A diffraction-limited focal spot corresponding to the targeted numerical aperture and the desired tilt-correction is achieved (Fig. S4 c). 


\section{Characterization of a focal spot size:}
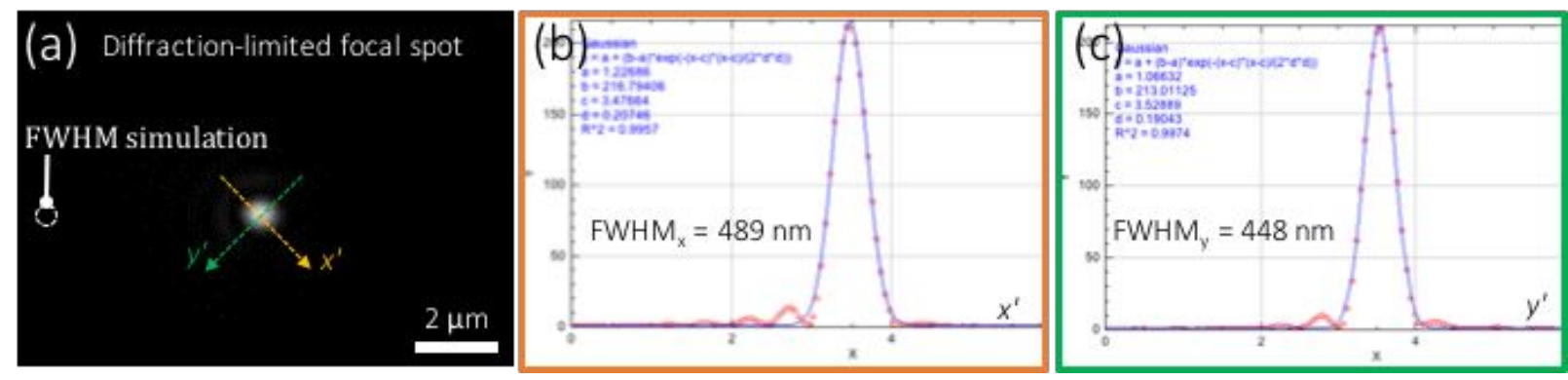

Fig. S5. Characterization of high-NA focusing of the photonic platform. (a) A focal spot pattern formed by $4.3^{\circ}$ angle-compensated MS mounted on the EMC projecting $\approx 195 \mu \mathrm{m}$ wide collimated Gaussian beam. The dashed circle indicates the FWHM of the diffraction-limited focal spot obtained using FDTD simulations. (b-c) Normalized intensity plot profiles (red circles) across the focal spot in panel (a) drawn along the $x^{\prime}$ and $y^{\prime}$ directions. The $x^{\prime}$ direction is collinear with the in-plane projection of the collimated free-space beam. Blue curves are Gaussian fittings. 
(a)

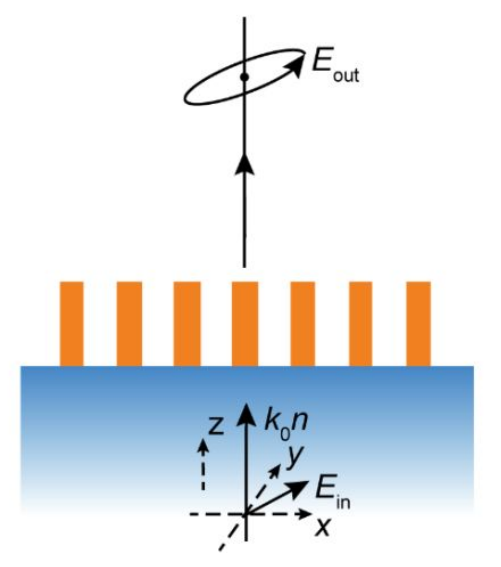

(b)

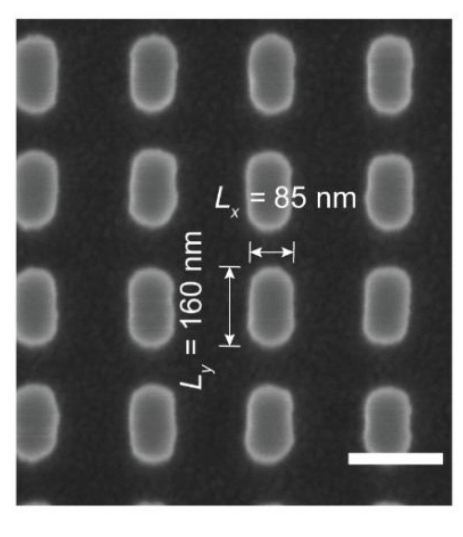

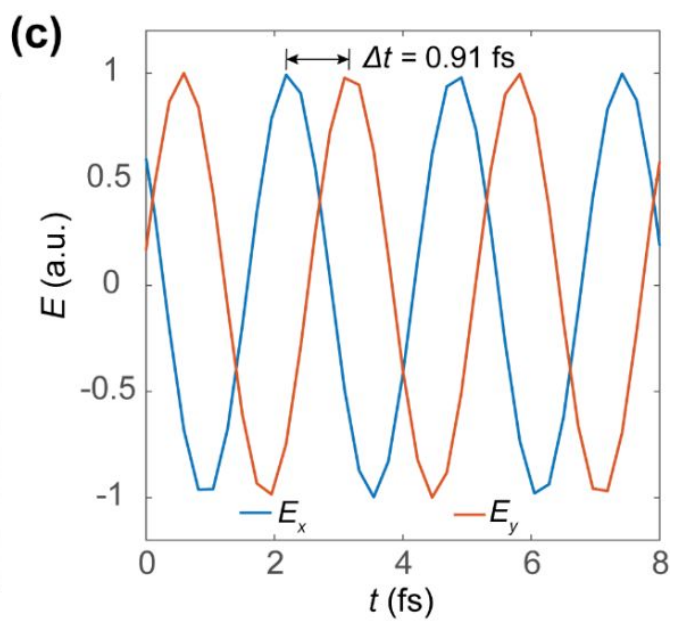

Fig. S6. Simulation of the polarization-conversion metasurface. (a) Schematic diagram of the metasurface functionality. (b) An SEM image of the fabricated metasurface characterized in Fig. 4. The in-plane dimensions, $L_{x}=85 \mathrm{~nm}$ and $L_{y}=160 \mathrm{~nm}$, are measured for FDTD simulations. A scale bar represents $200 \mathrm{~nm}$. (c) FDTD simulated $\boldsymbol{E}_{\boldsymbol{x}}$ and $\boldsymbol{E}_{\boldsymbol{y}}$ waveforms showing a time-delay of $0.91 \mathrm{fs}$.

The polarization conversion achieved in Fig. 4 is also confirmed by FDTD simulation. Here, the in-plane dimensions $\left(L_{x}=85 \mathrm{~nm}, L_{y}=160 \mathrm{~nm}\right)$ and height $(H=660 \mathrm{~nm})$ of the nanopillars are measured using scanning electron microscope (SEM) images. A linearly polarized light (electric field is $45^{\circ}$ from the $x$-axis) is incident from the substrate side of the metasurface (Fig. S6 a). The simulated polarization state of the beam exiting the metasurface is shown in Fig. S6 c, with $E_{x}$ delayed by $\Delta t=0.91 \mathrm{fs}$ (corresponding to a phase delay of $\delta=2 \pi c \Delta t / \lambda_{0}=0.7 \pi$ ) with respect to $E_{y}$, indicating an elliptically polarized light. The expected polarization contrast visibility is $e=0.51$, closely matching the experimentally measured value of $e=0.46 \pm 0.02$ in Fig. 4 . 